Res Publica. Revista de Historia de las Ideas Políticas

ISSN: 1576-4184

http://dx.doi.org/10.5209/RPUB.54997

\title{
Conflicto político y modelos contemporáneos de la democracia*
}

\author{
Pedro Rivas**
}

Recibido: 31 de marzo 2015 / Aceptado: 21 de octubre 2016

Resumen. Frente a los conflictos derivados de la violación persistente de derechos humanos y desde una comprensión de la democracia en la tradición de las más recientes teorías liberales de la justicia, se ha formulado una propuesta sedicente deliberativa. Por el contrario, en una tradición alejada de modelos liberales, se defiende un modelo agonista de democracia. La transición sudafricana, desde el apartheid a la democracia, ha sido terreno de polémica entre ambos modelos y sirve como campo de pruebas de su alcance. Por eso, en primer lugar, este trabajo presenta el papel desarrollado por la Truth and Reconciliation Commission, que guió dicha transición, para exponer después las propuestas de ambos modelos de democracia y la discusión generada entre ambos, y terminar planteando conclusiones más allá de los dos modelos.

Palabras clave: perdón político; agonismo; democracia deliberativa; justicia transicional.

\section{[en] Political Conflict and Contemporary Versions of Democracy}

\begin{abstract}
This paper analyses two opposing models of democracy -i.e. deliberative democracy and agonism. It draws conclusions on the shortcomings of each model, taking into account what might have been the consequences of applying them on the transition from the apartheid to democracy in South Africa. These models are opposed to each other in the way they sort out political conflicts emerged from lingering violations of human rights. Deliberative democracy comes from recent liberal theories of justice, while agonism defends an alternative model of democracy. The analysis is conducted from the role played by the Truth and Reconciliation Commission and the voices raised in the interpretation of this role by the defenders of each model.
\end{abstract}

Keywords: political forgiveness; agonism; deliberative democracy; transitional justice.

La manera de tratar los conflictos y desacuerdos en el interior de las comunidades políticas sigue siendo asunto principal de reflexión de cualquier teoría política contemporánea. Pero cuando tales conflictos alcanzan la gravedad derivada de la violación persistente de derechos humanos, nos encontramos ante casos paradigmáticos por su dificultad ${ }^{1}$. En este trabajo vamos a considerar dos intentos contemporáneos de resolver conflictos políticos. Para ello vamos a ver sus tesis frente a uno de los conflictos de especial gravedad más estudia-

* Este trabajo forma parte de los resultados del proyecto de investigación "Del discurso de la derrota al discurso del diálogo. Justicia transicional, memoria histórica y Constitución” dirigido por el Prof. Pedro Serna Bermúdez, subvencionado por el Ministerio de Educación y Ciencia (SEJ2007-64461/JURI).

** Universidad Austral, Argentina privas@ius.autral.edu.ar

1 Al punto de haberse desarrollado un cuerpo doctrinal que bajo la denominación de justicia transicional, ha intentado formular los principios necesarios para superar tales situaciones y reconducir a las comunidades que las han sufrido hacia un Estado de Derecho. 
do y discutido: la transición de Sudáfrica del régimen de apartheid a la situación actual. Desde una comprensión de la democracia en la tradición de las más recientes teorías liberales de la justicia, Amy Gutmann y Dennis Thompson han formulado una propuesta que denominan democracia deliberativa.

Por el contrario, en una tradición que se presenta en cierto modo alejada de tales modelos liberales, Chantal Mouffe defiende un modelo agonista de democracia. La transición sudafricana ha sido terreno de polémica entre ambos modelos y sirve como campo de pruebas del alcance de cada uno de ellos. De ahí que, en primer lugar, sea necesario hacerse cargo siquiera en lo esencial del papel desarrollado por la Truth and Reconciliation Commission que guió dicho proceso de transición. Después será el momento de exponer las propuestas de ambos modelos de democracia y la discusión generada entre ambos, para terminar planteando algunas conclusiones más allá de los dos modelos mencionados.

\section{Sentido y fines de la Truth and Reconciliation Commission}

Para hacerse cargo de la tarea de la Truth and Reconciliation Commission sirve acudir al Informe que editó la misma al finalizar su trabajo. Como señala la propia Truth and Reconciliation Commission, es en el Preámbulo de la Promotion of National Unity and Reconciliation Act de 1995 donde se proporciona la estructura dentro de la cual debe entenderse la creación y la tarea de la Commission. Y es que fue concebida como parte de un proceso de construcción de puentes diseñado para ayudar a guiar a la nación de un pasado de profundas divisiones a un futuro fundado en los derechos humanos y la democracia ${ }^{2}$.

Una de sus principales tareas fue desvelar todo lo posible la verdad sobre las graves violaciones a los derechos humanos, en la creencia de que tal tarea era necesaria para la promoción de la reconciliación y la unidad nacional. Al punto de que contar la verdad ayudaría, entre otras cosas, a restaurar la dignidad de las víctimas y a permitir a los victimarios enfrentarse plenamente con su propio pasado ${ }^{3}$. Para ello la propia Commission señaló diferencias específicas propias respecto a otras comisiones establecidas en otros países, tales como el poder de conceder amnistía a los victimarios considerados individualmente, la publicidad de sus procedimientos o las audiencias institucionales y especiales ${ }^{4}$.

En cuanto a sus fines, la Commission recibió cuatro tareas principales para lograr esos objetivos de unidad nacional y reconciliación. En primer lugar, analizar y describir las causas naturaleza y extensión de las graves violaciones a los derechos humanos. En segundo lugar, hacer recomendaciones al Presidente sobre las medidas para prevenir futuras violaciones a los derechos humanos. En tercer lugar, restaurar la dignidad cívica y humana de las víctimas a través del testimonio. Y, por último, otorgar amnistía a quienes hicieran revelación completa de hechos 5 .

Pero, la reconciliación no era solamente un fin, sino también un proceso. Y debía llevarse a cabo en diferentes niveles ${ }^{6}$.

\footnotetext{
Truth and Reconciliation Commission of South Africa Report, London, MacMillan, 1999, vol. 1, ch. 4, pars. 1-2. Ibidem, vol. 1, ch. 4, par. 3 .

Ibidem, vol. 1, ch. 4, pars. 25-28.

Ibidem, vol. 1, ch. 4, par. 32 .

Ibidem, vol. 1, ch. 5, pars. 12-13.
} 
En primer lugar, enfrentando la dolorosa verdad. Así, las revelaciones que obtuvo la Commission sirvieron para posibilitar exhumaciones y entierros que ayudaron a lograr paz para los familiares de las víctimas. La Truth and Reconciliation Commission reconoció que, sin embargo, la reconciliación de las víctimas con su propio dolor es un proceso profundamente personal, complejo e impredecible. Su trabajo mostró además las dificultades de los perpetradores de violaciones a los derechos humanos para asumir su propia culpa y vergüenza ${ }^{7}$. En segundo lugar, la Truth and Reconciliation Commission contribuyó en ocasiones a la reconciliación de las víctimas y los victimarios en casos específicos ${ }^{8}$. En un tercer sentido, ayudó al proceso de reconciliación entre las distintas comunidades que forman parte de la nación, especialmente entre grupos raciales y étnicos ${ }^{9}$. En cuarto lugar, promovió la reconciliación nacional, en la medida en que las personas se perdonan entre sí y trabajan juntos como nación ${ }^{10}$. Por último, y con menos relevancia para nuestro propósito, suscitó la necesidad de la redistribución frente al legado de graves desigualdades sociales que produjo el apartheid ${ }^{11}$.

Ahora bien, para lograr el perdón, la Truth and Reconciliation Commission consideró necesario dar a las víctimas el necesario espacio para su dolor dando voz a los sentimientos previamente silenciados. Al mismo tiempo, muchas de las víctimas mostraron una extraordinaria magnanimidad y generosidad de espíritu mediante su disposición a perdonar ${ }^{12}$. Todo ello no supuso olvidar sino buscar superar la amargura y renunciar al resentimiento. Y así, al revelar la verdad, se evitó la tentación de recordar de modo partidista y selectivo. Una memoria que incluya la verdad dolorosa fue necesaria para lograr superar las divisiones del pasado ${ }^{13}$.

La Truth and Reconciliation Commission fue consciente de las tensiones entre amnistía, verdad y justicia. Pero indica que el mandato constitucional recibido era el de la necesidad de entendimiento y no de venganza, de reparación y no de represalia, de $u b u n t u^{14}$ y no de victimización. Un compromiso que incluía el fortalecimiento de las dimensiones restaurativas de la justicia. Esta última es un proceso que, entre otras cosas, se basa en la reparación porque busca sanar y restablecer a las víctimas en primer lugar, pero también a los victimarios, a las familias y a toda la comunidad. Y lo hace, fomentando que todos estos actores se involucren ${ }^{15}$. Para ello ha acudido a las dimensiones restaurativas que se encuentran en distintas tradiciones que existen en Sudáfrica. En concreto, la judeocristiana y los valores tradicionales africanos porque ambas contienen fuertes fuentes de curación y restablecimiento comunitarios ${ }^{16}$.

\footnotetext{
Ibidem, vol. 1, ch. 5, pars. 14-15.

Ibidem, vol. 1, ch. 5, par. 16.

Ibidem, vol. 1, ch. 5, par. 17.

Ibidem, vol. 1, ch. 5, par. 23.

Ibidem, vol. 1, ch. 5, pars. 24-28.

Ibidem, vol. 1, ch. 5, pars. 49-50.

Ibidem, vol. 1, ch. 5, pars. 50-51.

La propia Truth and Reconciliation Commission define Ubuntu como "humanidad" (humanness), señalando que se manifiesta metafóricamente en la expresión "las personas son personas a través de otras personas" (people are people through other people), ibidem, vol. 1, ch. 5, par. 85.

Ibidem, vol. 1, ch. 5, par. 82 .

Ibidem, vol. 1, ch. 5, par. 84 .
} 


\section{Conflicto y deliberación}

Gutmann y Thompson han formulado los fundamentos de su versión de la democracia deliberativa a la par que trataban de mostrar el aporte de la misma a las situaciones de conflicto características de la justicia transicional. De hecho sus tesis sobre el fundamento moral de las comisiones de la verdad ${ }^{17}$ están escritas en medio del debate que generó su principal obra, Democracy and Disagreement ${ }^{18}$, y antes de otras en las que han seguido desarrollando su pensamiento ${ }^{19}$. De ahí que sean los referentes inevitables en el presente debate.

Hay que comenzar por indicar que la perspectiva que defienden Gutmann y Thompson pretende ser una corrección a la par que una profundización del planteamiento rawlsiano. La pregunta que se plantean es qué género de democracia es mejor para vivir con desacuerdos morales, es decir, para lograr una perspectiva que pueda ser aceptada por gente mutuamente motivada a encontrar términos justos de cooperación social entre personas políticamente iguales ${ }^{20}$. A su juicio, dos son los modelos de democracia relevantes en el momento presente. Por un lado, la que denominan democracia procedimental, según la cual lo importante es establecer procesos legítimos y justos para construir decisiones sobre temas morales controvertidos. Tales procesos requieren de la regla de las mayorías y de los derechos individuales necesarios. No se trata de que la mayoría tenga ea ipsa la razón sino de que constituye el procedimiento más equitativo. Con la referencia a los derechos que intervienen en el procedimiento democrático se refieren a aquellos como las libertades de opinión, asociación, información, sufragio, etc., es decir solamente a aquellos cuyo fin primario es hacer justo el procedimiento ${ }^{21}$. Por el otro, la que denominan democracia constitucional (sostenida por Rawls) que añadiría otros derechos como prioritarios aunque no aparezcan en el procedimiento de decisión. A juicio de los defensores de ésta, hay que tener en cuenta más libertades básicas y, en especial, la defensa de la igualdad de oportunidades, que no se pueden someter a la regla de las mayorías. Ambas perspectivas coinciden en defender la necesidad de procedimientos moralmente justificados para llegar a decisiones políticas vinculantes ${ }^{22}$. En opinión de Gutmann y Thompson, la democracia deliberativa que ellos defienden trata de evitar esa dicotomía entre procedimiento y derechos, porque resulta incapaz de resolver los desacuerdos morales.

En el caso de Rawls parece que los desacuerdos se superan, pero se trataría de una percepción errada producto de que permanece siempre en términos demasia-

17 Amy Gutmann \& Dennis Thompson, “The Moral Foundations of Truth Commissions", en Robert I. Rotberg \& Dennis Thompson (eds.), Truth v. Justice: The Morality of Truth Commissions, Princeton, Princeton University Press, 2000, pp. 22-44.

18 Amy Gutmann \& Dennis Thompson, Democracy and Disagreement. Why moral conflicts cannot be avoided in politics, and what should be done about it, Cambridge, Harvard University Press, 1996. Es interesante a este respecto hacer énfasis en el subtítulo de esta obra. También "Democratic Disagreement" en Stephen Macedo, Deliberative Politics. Essays on Democracy and Disagreement, Oxford/New York, Oxford University Press, 1999, pp. 243-279.

19 Amy Gutmann \& Dennis Thompson, Why Deliberative Democracy, Princeton, Princeton University Press, 2004 y Amy Gutmann \& Dennis Thompson, The Spirit of Compromise. Why governing demands it and campaigning undermines it, Princeton, Princeton University Press, 2012.

20 Amy Gutmann \& Dennis Thompson, "The Moral Foundations of Truth Commissions", op. cit., p. 26.

$21 \quad$ Ibidem, pp. 27-32.

22 Ibidem, pp. 33-34. 
do abstractos. Para Rawls, la indeterminación de su teoría de la justicia comienza cuando termina la reflexión solipsista bajo el velo de la ignorancia ${ }^{23}$. Ahora bien, cabe preguntarse qué políticas satisfarían el principio de diferencia o la igualdad de oportunidades ${ }^{24}$. En el primer caso y ciñéndose al ejemplo de la salud se puede señalar la compleja discusión sobre los tan distintos sistemas de acceso a la misma. En el segundo, el ámbito de las políticas de discriminación positiva es muestra del profundo desacuerdo social sobre el mismo ${ }^{25}$.

Con todo, es en el propio Rawls donde puede encontrarse el inicio de un modo de evitar estas aporías. Por un lado, porque incorpora la necesidad de valores ciudadanos como los de superar el propio interés y de guiarse por alguna concepción de la justicia y del bien común más que por sus propias inclinaciones ${ }^{26}$. Por el otro, con su énfasis en el papel de la reciprocidad como guía de la discusión pública, que conduce a una idea de la razón pública que contiene argumentos morales que deben ser consistentes con dicha reciprocidad ${ }^{27}$. Y en tercer lugar, porque la perspectiva deliberativa se parece al consenso entrecruzado o superpuesto de Rawls, en la medida en que ambos quieren proveer un punto desde el que juzgar pretensiones morales enfrentadas de la forma más independiente posible de visiones morales comprehensivas ${ }^{28}$.

La propuesta de una democracia deliberativa viene a decir que las decisiones colectivas son más legítimas en la medida en que sean el resultado de la discusión pública entre sujetos libres e iguales ${ }^{29}$. Los participantes son llamados a justificar sus preferencias políticas en términos que puedan ser aceptadas razonablemente por aquellos a quienes les afectan. De este modo, los ciudadanos no pueden limitarse únicamente a hacer valer sus intereses particulares sino que han de ser capaces también de formularlos en términos de principios morales generales con los que todos puedan estar potencialmente de acuerdo ${ }^{30}$. La deliberación se orienta, entonces, hacia el logro del consenso, aunque éste rara vez se alcance en la práctica ${ }^{31}$. Por eso, a pesar de que finalmente el desacuerdo conduzca a una elección mayoritaria, la decisión será más legítima, $\mathrm{y}$, por tanto, moralmente más vinculante para la minoría, en la medida en que las perspectivas, valores e intereses de todos los afectados por la decisión hayan sido justamente representados y tomados en cuenta. Sólo presuponiendo la posibilidad de llegar a un consenso, el conflicto y el desacuerdo pueden ser manejados dentro de un horizonte de sentido compartido por las partes en conflicto ${ }^{32}$.

El valor fundamental, donde descansa su teoría de la democracia deliberativa, es la reciprocidad. Ésta significa que los ciudadanos (i) buscan términos justos de cooperación social ${ }^{33}$, al tiempo que (ii) son capaces de justificar sus ideas políticas y (iii) de tratar con respeto a quienes se esfuerzan por comprometerse en esa tarea común, incluso si no logran soluciones ${ }^{34}$. Es decir, que deben dar no sólo razones contingen-

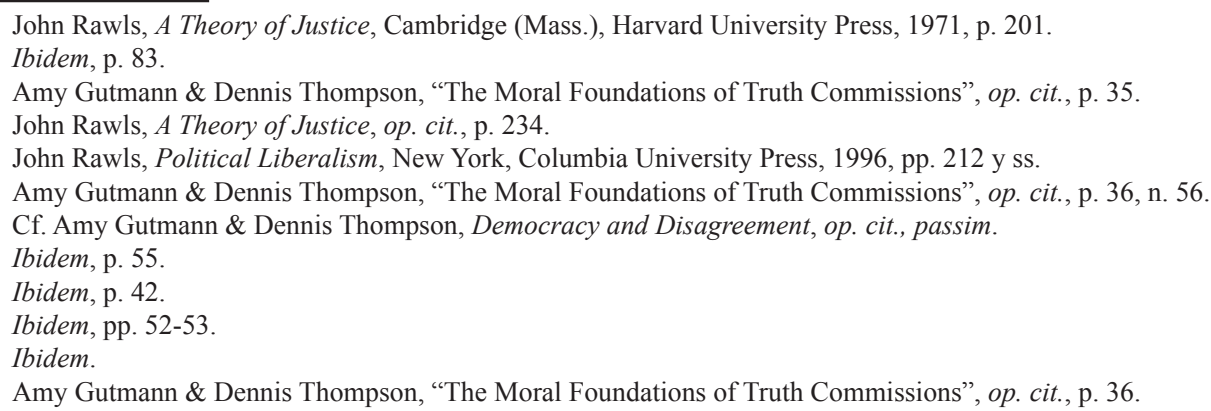


tes basadas en sus intereses, sino razones morales generales por las que aquellos con quienes se está en desacuerdo deberían aceptar los resultados de la negociación ${ }^{35}$.

La reciprocidad se compara con otros dos principios posibles para manejar el desacuerdo. Por un lado, el principio de prudencia, que busca el acuerdo entre intereses de grupo o de clase, y que resulta, por tanto, menos exigente que el de reciprocidad. Por el otro, está el principio de imparcialidad, que pide razonar en términos morales universales, y que, en la medida en que comprende el altruismo, es más exigente que la mera reciprocidad. Sin embargo, la reciprocidad no exige de los participantes imparcialidad ni altruismo. Los ciudadanos no tienen que trascender su propio interés sino sólo representar (presentar, expresar, manifestar, formular) sus posiciones particulares en términos de principios generales que otros puedan razonablemente $\operatorname{aceptar}^{36}$.

A su juicio, éste es el mejor marco posible para justificar el trabajo de las comisiones de verdad frente a sociedades divididas, en la medida en que su modelo afronta controversias que, en último término, son morales ${ }^{37}$. Más en concreto, el fundamento de las comisiones para la verdad y la reconciliación no es simplemente el intento de alcanzar un pacto entre las partes enfrentadas para evitar de este modo un final violento al desacuerdo. Si así fuera, careceríamos de buenas razones para valorar la estabilidad política por encima de otros valores posibles como, por ejemplo y especialmente, la justicia ${ }^{38}$.

Se hace necesario acudir a los principios que sostienen las condiciones de la discusión política. Y ahí, ante la falta de consenso propia de todo conflicto, la reciprocidad democrática exige buscar una economía del desacuerdo moral. Las posiciones frente al otro se justifican mediante la búsqueda de un rationale que minimice los rasgos de la posición contraria que uno rechaza. Siguiendo este principio, los ciudadanos buscan puntos significativos de convergencia entre las distintas comprensiones. Al economizar así los desacuerdos, los ciudadanos manifiestan respeto mutuo aunque continúan en desacuerdo sobre aspectos moralmente importantes sobre los que deben alcanzar una decisión colectiva. Se contribuye de esta forma a una política más efectiva y realista porque los distintos actores buscan una base común y aprenden a respetar las divisiones que permanecen. Solamente queda excluido de esta convergencia quienes no están dispuestos a la reciprocidad ${ }^{39}$. Para alcanzar tales puntos de convergencia, se hace necesario evitar razones y argumentos fundados en doctrinas omnicomprehensivas del bien. Tampoco se trataría de intentar que todos actúen por altruismo, como en el caso del perdón cristiano, que orientó el trabajo de la sudafricana Truth and Reconciliation Commission, y que presupone además una doctrina comprehensiva del bien ${ }^{40}$.

Así, el principio de reciprocidad otorga una perspectiva integradora que permite un margen amplio de desacuerdo razonable ${ }^{41}$. Reconocen, eso sí, que la presencia de la razonabilidad en su propuesta es un mecanismo de exclusión, aunque sirve para justificar moralmente que quienes no buscan esos términos justos de cooperación so-

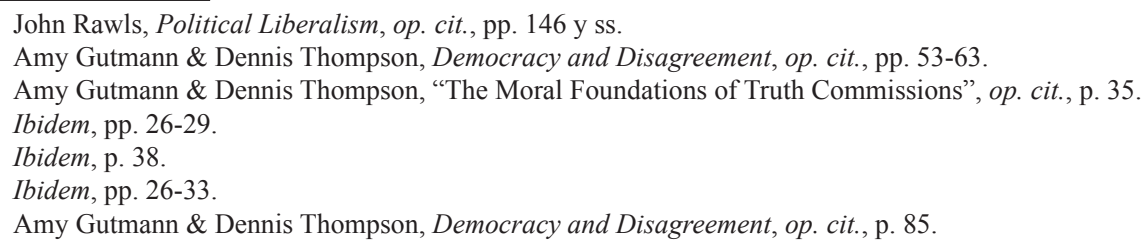


cial quedan fuera de la discusión ${ }^{42}$. Una perspectiva moral compartida sólo es posible si hay criterios de distinción entre lo razonable y lo irrazonable ${ }^{43}$. Así, por ejemplo, en el caso sudafricano, sería irrazonable tratar de negar la involucración de las instituciones políticas en los crímenes que se habían cometido ${ }^{44}$.

Con su propuesta, como ya se ha dicho, no se haría necesario buscar la reconciliación a través del perdón. El principio de economía moral aspira a lograr un cierto grado de respeto entre los ciudadanos. No requiere intimidad ni cercanía con el otro. Resulta suficiente que los distintos actores del conflicto se respeten entre sí y se reconozcan el uno al otro como miembros de la comunidad política y tengan la voluntad de tratarse entre ellos como tales ${ }^{45}$. Es decir, no hace falta más que reconocer sinceridad y compromiso en el otro para lograr términos equitativos de cooperación social ${ }^{46}$. Con todo, aunque no exija el perdón y la benevolencia, sí es capaz de acomodarlo porque la perspectiva de la democracia deliberativa respeta todas las respuestas. Igualmente, el principio de economía moral no requiere un consenso conclusivo y completo acerca de los hechos pasados. La práctica de los testimonios típica de las comisiones de la verdad supone hacer real esa reciprocidad en la medida en que necesita de la apertura mental a considerar lo que otros relatos. Aun así, el principio de economía moral no significa imparcialidad moral. Al contrario, es una práctica moral, gobernada por principios morales ${ }^{47}$.

\section{Conflicto y agonismo}

Desde un punto de vista opuesto, encontramos otro intento de explicar y resolver los conflictos políticos en los defensores de la denominada democracia agonista. Para Chantal Mouffe, referente de esta perspectiva, ésta es más bien un ethos que afirma la contingencia y apertura de la vida política. El "nosotros" político es un logro difícil, frágil y contingente.

Mouffe define lo político como la dimensión de antagonismo constitutivo de las sociedades humanas, frente a la política que sería el conjunto de prácticas e instituciones a través de las cuales se crea un determinado orden que organiza la coexistencia humana en el contexto de la conflictividad ${ }^{48}$. El problema central del liberalismo radica precisamente en la negación del carácter irreductible del antagonismo ${ }^{49}$.

Su caracterización de lo político y la consiguiente crítica al planteamiento liberal hunde sus raíces en las tesis de Carl Schmitt. Para éste, lo político sólo se entiende en la agrupación amigo/enemigo. El racionalismo liberal no sería capaz de entender que sus pretendidos consensos se basan en actos de exclusión, de modo que el elemento central de lo político es el del antagonismo que hace presente ese límite del consenso racional. Mouffe comenta al respecto que, en efecto, el nosotros, la forma colectiva

\footnotetext{
$42 \quad$ Ibidem, p. 55.

43 Amy Gutmann \& Dennis Thompson, "Democratic Disagreement" en Stephen Macedo, Deliberative Politics. Essays on Democracy and Disagreement, op. cit., pp. 257-259.

44 Amy Gutmann \& Dennis Thompson, "The Moral Foundations of Truth Commissions", op. cit., p. 37.

45 Ibidem, p. 39.

$46 \quad$ Ibidem, pp. 37-38.

47 Ibidem, pp. 40-41.

48 Chantal Mouffe, On the Political, London \& New York, Routledge, 2005. Empleo la traducción castellana de Soledad Laclau, En torno a lo político, Buenos Aires, Fondo de Cultura Económica, 2007, p. 16.

49 Ibidem, p. 19.
} 
de identificación, tiene que ver con el conflicto ${ }^{50}$ y el antagonismo, porque las identidades políticas son del tipo nosotros/ellos ${ }^{51}$.

La diferencia central con Schmitt es que Mouffe pretende compatibilizar el planteamiento recién expuesto con el reconocimiento del pluralismo, que es constitutivo de la democracia moderna. Para esto, es necesario reconocer primero que el antagonismo permanece siempre como una posibilidad y que no hay un fundamento último del orden político ${ }^{52}$. Es decir, que el poder es constitutivo de las relaciones sociales, al punto que la objetividad social es en última instancia política y muestra necesariamente las huellas de una exclusión. El poder no es una relación externa entre identidades preconstituidas sino que él mismo constituye las identidades ${ }^{53} \mathrm{y}$, por eso mismo, las identidades tampoco son estables sino resultado de procesos de identificación ${ }^{54}$. De este modo, el poder crea las relaciones sociales primarias sin condiciones racionales previas ${ }^{55}$. Por eso, todo orden social es hegemónico y se basa, por tanto, en alguna forma de exclusión. Y las prácticas que establecen ese orden y que dan sentido a las instituciones sociales son prácticas hegemónicas ${ }^{56}$. Democracia significará solamente que ningún actor social puede atribuirse la representación de la totalidad ni pretender ser el dueño. Esa naturaleza puramente construida de las relaciones sociales se complementa con los fundamentos puramente pragmáticos de la legitimidad del poder. No porque el poder sea automáticamente legítimo sino porque todo poder se impone porque es reconocido legítimo hasta cierto punto. La legitimidad se basa en alguna forma de poder exitoso no en argumentos racionales ${ }^{57}$.

Con todo, el problema permanece sin resolver: ¿cómo es posible compatibilizar antagonismo y pluralidad democrática ${ }^{58}$, poder y valores democráticos ${ }^{59}$ ? Para lograrlo, habrá que distender o domesticar el antagonismo, transformarlo en agonismo para que no destruya la comunidad política ${ }^{60}$. Es decir, hará falta una relación agónica donde, sin verse como enemigos, las partes reconocen que no existe solución racional al conflicto. Para tal cosa, reconocen también la legitimidad de sus oponentes, o sea su común pertenencia a la misma asociación política. El conflicto entre proyectos hegemónicos opuestos se canaliza para que no derive en violencia, por ejemplo a través del sistema parlamentario, que no es negociador ni deliberativo sino agonista ${ }^{61}$.

Se hace necesario tomar conciencia de que lo que sustentan dichos proyectos hegemónicos son pasiones y que lo importante es darles una salida democrática ${ }^{62}$. Se tratará de no ver al otro como enemigo a destruir sino como adversario, es decir, como aquel al que se le respeta su derecho a contestar los términos de nuestra asocia-

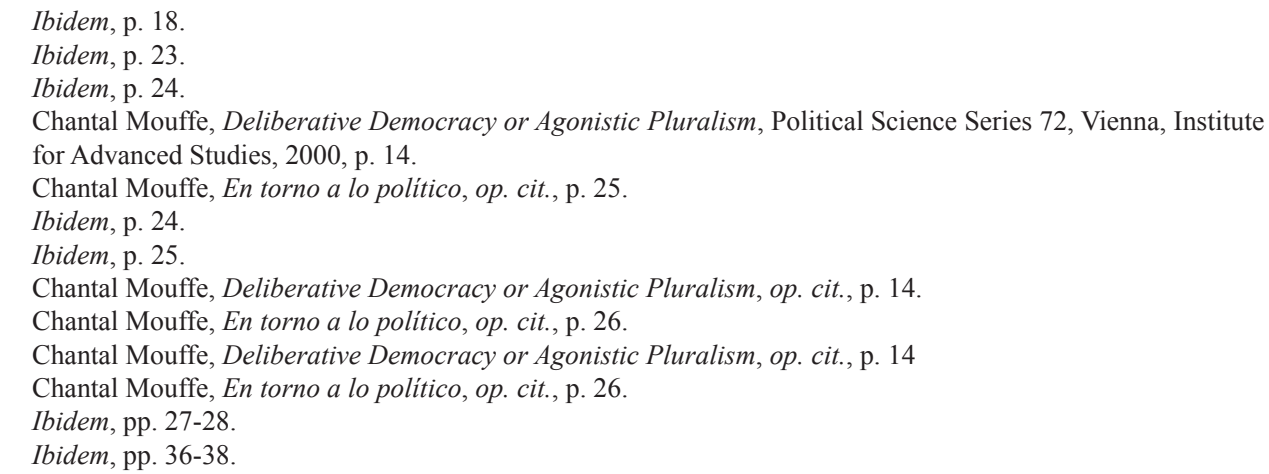


ción política ${ }^{63}$. Pero el respeto tiene presente que, como se ha dicho, no hay solución racional accesible para el conflicto ${ }^{64}$.

Con todo, Mouffe afirma que no busca una refundación radical del orden social ni rechaza el marco de la democracia liberal. Es más, parte igualmente del consenso básico en las instituciones constitutivas de la democracia y en los valores éticos y políticos que la inspiran, es decir, en la libertad y la igualdad ${ }^{65}$.

Desde aquí se entiende su crítica al planteamiento deliberativo. En efecto, Mouffe sostiene que los defensores de la democracia deliberativa se equivocan porque no tienen en cuenta la necesidad de un consenso moral previo sobre los términos en los que el conflicto cobra sentido: y este consenso se constituye siempre políticamente. Lo político empieza antes, se discute siempre en una forma de vida, en una tradición compartida. Si hay conflicto político, es que entonces no hay siquiera esa forma de vida, esa tradición: hay más bien un conflicto entre identidades políticas, no entre doctrinas comprehensivas razonables ${ }^{66}$.

Aplicando estas tesis a las sociedades divididas, Schaap sostiene que en realidad en tales situaciones conviven varias tradiciones y es la hegemónica la que establece los términos de juego. El consenso no se producirá a partir de nada común, sino que será en todo caso un resultado contingente de la interacción política. Por eso, los que no pertenecen a la tradición dominante aparecen como poco razonables, de forma que la tradición dominante termina por neutralizarles ${ }^{67}$. De ahí su crítica al planteamiento de Gutmann y Thompson. En efecto, en una sociedad dividida falta precisamente lo que ellos dan por supuesto: respeto, reconocimiento del otro, reciprocidad. El conflicto es más radical, es de identidades ${ }^{68}$. Apuesta, por el contrario, por una democracia agonista que no cree en el consenso ni en que las partes en conflicto se acerquen o cambien ${ }^{69}$.

No es este el lugar para tratar en detalle el pensamiento político de Mouffe, aunque lo que se diga sobre su operatividad en el terreno de la justicia transicional signifique ya una cierta valoración del mismo. Con todo, salta a la vista que su planteamiento presenta varios problemas. Por mencionar algunos, puede señalarse en primer lugar que su propuesta necesita hacer espacio a quienes critica. En efecto, como Rawls, requiere de acuerdos fundamentales sobre valores nucleares y procedimientos que regulen, canalicen y legitimen los conflictos políticos. Igualmente necesita del respeto entre ciudadanos y del derecho de disenso. E incide en la búsqueda de una voz política para los grupos más vulnerables. En definitiva, que sus tesis descansan implícitamente en el intercambio equitativo de razones entre iguales, característico de aquellos que critica ${ }^{70}$. Y la con la diferencia añadida de que en su caso no hay intento de justificar todos esos implícitos.

En segundo lugar, la visión agonista de Mouffe supone una incesante confrontación con las hegemonías establecidas, sin poner en riesgo los términos de la comu-

\footnotetext{
Chantal Mouffe, The Democratic Paradox, London, Verso, 2000, p. 101.

Ibidem, p. 102

Chantal Mouffe, En torno a lo político, op. cit., pp. 38-40.

Chantal Mouffe, The Democratic Paradox, op. cit., pp. 26-32.

67 Andrew Schaap, “Agonism in divided societies": Philosophy \& Social Criticism, vol. 32 (2006), pp. 255-277, aquí p. 263.

68 Ibidem, p. 266.

69 Ibidem, pp. 269-272.

70 Keith Breen "Agonism, Antagonism and the Necessity of Care", en Andrew Schaap (ed.), Law and Agonistic Politics, Farnham, Ashgate, 2009, pp. 133-146, aquí p. 138.
} 
nidad política. Ahora bien, esto resulta contradictorio porque esos términos o condiciones han sido precisamente impuestos por los grupos hegemónicos para desplazar al resto del espacio público común ${ }^{71}$. Por tanto, sólo cabe contestar también los mismos términos de la comunidad o aceptar que no todas las hegemonías establecidas pueden confrontarse.

Además, su idea de que una política agonista mantiene bajo control el antagonismo y posee también un efecto integrador, resulta cuando menos optimista sino más bien ingenua. Para sostener tal cosa tiene que dar por supuesto que lo ámbitos de discusión son pequeños, lo cual no coincide ni con su descripción de las situaciones de hegemonía ni con gran parte de la realidad presente ${ }^{72}$.

Pero, más allá de los problemas generales, si se aplica su propuesta a los conflictos característicos de las situaciones de justicia transicional, seguimos encontrando numerosas críticas. Dryzek, por ejemplo, considera que la perspectiva agonista tiene algunos problemas. Por un lado, no está claro cómo y por qué los ciudadanos de una sociedad dividida que se enfrenta a un proceso transicional se mostrarán un respeto capaz de mantener el conflicto alejado de la violencia, tal y como lo pretende el planteamiento agonista. Es más en estas situaciones se puede reproducir el mismo argumento que desde la teoría agonista se empleaba contra los defensores de la democracia deliberativa, es decir, que en situaciones de conflicto lo que falta es la aceptación mutua de razonabilidad y por tanto no es esperable encontrar razones para el respeto. Además, los teóricos de la democracia deliberativa proponen cuando menos un procedimiento para regular la confrontación política, aspecto este que está ausente en la perspectiva de la democracia agonista ${ }^{73}$.

Pero es que lo que parece esperable que se produzca con un enfoque agonista es justo lo que se pretende evitar, pues, en efecto, es de imaginar que las identidades en conflicto se fortalecerán en lugar de transformarse. De esta forma nos alejamos de una posible solución no violenta ${ }^{74}$.

Pero las críticas no han venido solamente desde fuera. Aun siendo crítico de las tesis de la democracia deliberativa y a pesar de apreciar las tesis del agonismo porque recuerdan la fragilidad de lo político y por su dosis de realismo al mostrar los riesgos de la irreversibilidad de los conflictos, sin embargo Schaap encuentra numerosos problemas en el planteamiento de Mouffe $^{75}$. A su juicio, ésta no puede lograr un uso del concepto de lo político de Schmitt que sea compatible con el pluralismo democrático, porque el conflicto aparece siempre como una dicotomía en la que no se ve la forma de hacer surgir un tercero ${ }^{76}$. En especial, porque la presunción de Mouffe de que el conflicto necesariamente tiene una función integradora dentro de una política democrática no está garantizada en modo alguno. Y lo mismo puede decirse de su presunción más básica, la de que cada identidad política se forma necesariamente en oposición a un antagonista ${ }^{77}$.

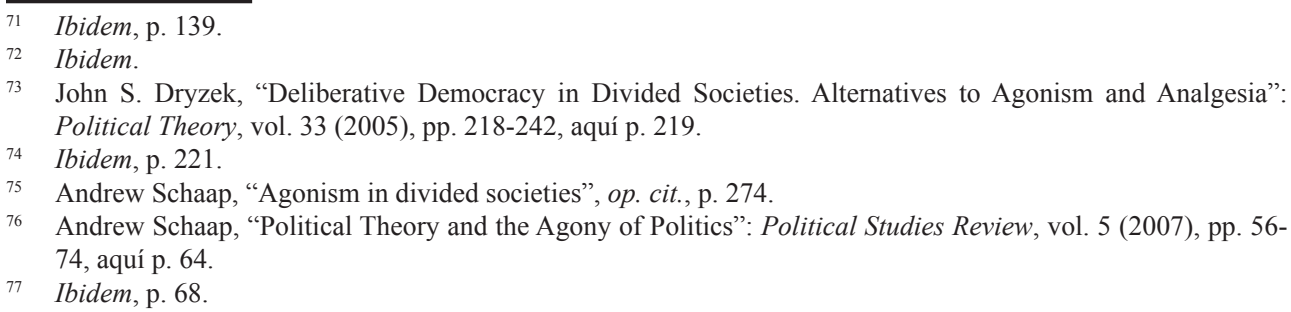


Más en general, sostiene que las tesis de Mouffe carecen de una adecuada institucionalización de la democracia agonista y de este modo no consiguen conformar una alternativa real ${ }^{78}$.

\section{Consecuencias para la Filosofía Política}

Vistos los términos de la discusión presente, corresponde intentar terciar en ella sin perder de vista que el foco está puesto en la justicia transicional. Tratar de resolver los fundamentos de la teoría política al hilo de la cuestión de este trabajo sería imposible además de pretencioso. Ahora bien, a mi juicio, la situación de transición del régimen sudafricano del apartheid a un verdadero estado democrático de Derecho pone de manifiesto la inanidad de las teorías de la democracia deliberativa. Sin compartir la visión agonista, hay que dar la razón a quienes muestran la incapacidad de construir una comunidad política después del apartheid a partir de los principios formulados por Gutmann y Thompson. En efecto, no sólo es que no hay un punto de partida común, una identidad compartida que posibilite exponer razones morales generales y alcanzar un fundamento racional que minimice el rechazo de las posiciones minoritarias. A mi juicio, hace falta recordar un hecho fundamental. La peculiaridad del apartheid radica, para el caso que nos ocupa, en que una enorme mayoría de la población blanca o bien apoyó o bien no hizo nada por terminar con el régimen del apartheid. Así, el partido anti-apartheid más característico tuvo un único diputado durante muchos años y logró su mejor resultado en las elecciones parlamentarias de 1981 y 1989 , en las que apenas alcanzó el 20\%. Por señalar otro ejemplo de lo que se viene diciendo, Dyzenhaus ha mostrado cómo sentencias que aplicaban leyes características del régimen del apartheid fueron dictadas por jueces de todas las ideologías políticas ${ }^{79}$. En definitiva, la oposición y resistencia, incluso la mera crítica, entre la población blanca al apartheid fue minoritaria y fácilmente individualizable. Esto distingue el régimen del apartheid de otras situaciones de transición. Para los blancos no se trataba de un régimen de terror ni la crítica operó para ellos como motivo suficiente de su eventual eliminación física o de su encarcelación como disidentes. Tampoco se trataba de un régimen que tuviera recursos para ocultar la realidad a sus propios ciudadanos, ni que obligara a la colaboración bajo amenazas de daños graves para uno mismo o para sus familiares. Ni se estaba ante el final de una guerra, durante la cual la debilidad del estado de Derecho hubiese sido aprovechada en ambos bandos por algunos para cometer impunemente crímenes, sin que los ciudadanos comunes pudiesen considerarse responsables siquiera indirectos de tales crímenes, al no tener conocimiento de ellos o al estar movilizados militarmente.

Ante esta situación, ¿qué sentido tiene hablar de consenso, deliberación pública, términos justos de cooperación social, reciprocidad, empleo de razones morales generales, búsqueda de un fundamento que minimice el rechazo de la posición contraria, intento de hallar puntos de acuerdo ajenos a una doctrina comprehensiva común, reconocimiento de sinceridad y compromiso en el otro para lograr términos justos (equitativos) de cooperación social? No se pretende ahora defender la venganza, sino

Ibidem, pp. 69-72.

79 Cf. David Dyzenhaus, Judging the Judges, Judging Ourselves. Truth, Reconciliation and the Apartheid Legal Order, Oxford, Hart, 1998, especialmente el capítulo 2. 
de poner de relieve que la población negra no tenía ninguna razón para esperar esto de la mayoría de la población blanca. Y aunque la tuviera, la pregunta permanece: ¿por qué la población negra habría de querer construir una comunidad política con la mayoría de la población blanca, la misma que mantuvo o permitió el apartheid? Dicho de otro modo: ¿es razonable formar parte de la misma comunidad política con aquellos que, sin sufrir coacción alguna, han permitido los crímenes del apartheid? Para perseguir un consenso se requiere que el otro sea capaz de perseguirlo. Pero, ¿no es claro que, durante los años del apartheid, la mayoría de la población blanca no quería ningún consenso con quien pertenecía a otra raza? ¿Cómo reconocer sinceridad y compromiso en quien apoyó o permitió con total espontaneidad el régimen del apartheid?

A mi modo de ver, esta situación sólo podía resolverse coherentemente de dos maneras. La primera, por lo visto hasta aquí, con la expulsión de Sudáfrica de esa mayoría de la población blanca. La segunda, con la solución que aportó la Truth and Reconciliation Commission. Esta fue completamente contraria a la pretendida desde los principios de la democracia deliberativa, pues se basó precisamente y de modo explícito en una doctrina comprehensiva del bien. En este sentido, hay que dar la razón a las críticas que desde un planteamiento agonista se hacen al ideal de democracia deliberativa. Y no dársela cuando sus críticas se dirigen a la actuación de la Truth and Reconciliation Commission. Porque si no cabe otra alternativa posible a la que se ha planteado aquí, lo único coherente desde la democracia agonista sería reconocer y defender la opción de la expulsión de los blancos del país. La alternativa que se presenta no es el fruto de una exageración sino la consecuencia inmediata de considerar la situación política de la transición sudafricana. Es evidente que las cosas podían haberse hecho de maneras diversas a las que plantea esta disyuntiva, pero en ningún caso se trataría de soluciones coherentes. Además, el proceso requirió llevar a cabo la adopción de un conjunto de valores que va mucho más lejos de lo que pretende el ideal deliberativo. La Truth and Reconciliation Commission formuló esos valores y apeló a ellos con toda claridad cuando señaló cuáles eran los fines que perseguía y no tuvo inconveniente en señalar que se trataba de bienes comprensibles desde una concreta tradición religiosa (la judeocristiana) y desde una tradición cultural particular (Ubuntu) ${ }^{80}$.

Hasta aquí podría dar la impresión de que la excepcionalidad de la situación sudafricana, con esa peculiaridad del apartheid a la que se ha hecho referencia, convierte la conclusión alcanzada en un caso tan particular que no puede extenderse al resto de transiciones políticas. Sin embargo, este carácter peculiar no debe hacer olvidar algo común: el carácter trágico del pasado político. Cada tragedia tiene sus condiciones distintivas (en este caso, qué papel desempeñaron los victimarios), pero todas coinciden en unos hechos pasados penosos y amargos. Esta realidad no se da sólo en los procesos más recientes (las transiciones de la Europa del Este o de Iberoamérica, por ejemplo), sino también en otras más lejanas: desde las revoluciones del siglo XVIII hasta la Transición española, pasando por las revoluciones nacionales del XIX (por citar solamente algunos casos), encontramos siempre situaciones precedentes de guerras, luchas y autoritarismo de diversa intensidad y sentido. Sin necesidad de que las dimensiones de los hechos trágicos sean comparables, en todas estas situaciones hay conciencia de salir de un pasado doloroso y de construir una realidad política

80 Truth and Reconciliation Commission of South Africa Report, vol. 1, ch. 5, par. 84. 
diferente. Dicho de otro modo, ese carácter trágico común pone de manifiesto el carácter fundacional, originario, inaugural. Esta experiencia ha sido vivida por cada comunidad política presente al menos una vez en su historia y en muchos casos, varias veces. Es decir, no se trata de una excepción sino de algo relativamente frecuente en la historia de las naciones. Y es precisamente en esos momentos fundacionales cuando la comunidad política se define realmente a sí misma. Por eso, la manera de definirse no es nunca abstracta ni consiste en encontrar puntos de acuerdo al margen de una doctrina comprehensiva. Al contrario, consiste en formular aquellos bienes que se desea estén presentes en el nuevo orden político y lo caractericen por contraste con los hechos del pasado inmediato que se intenta dejar atrás. ¿Dónde si no se encuentra, por ejemplo, el sentido de la Primera Enmienda de la Constitución americana, el del artículo $1^{\circ}$ de la Ley Fundamental de Bonn o la inclusión del pluralismo político como valor superior en la Constitución española? En el caso que nos ocupa, la Truth and Reconciliation Commission no hizo sino obedecer un mandato constitucional. En cierto sentido, algo de esto anticipa Schaap al comprobar que los problemas de las teorías mencionadas (democracia deliberativa y democracia agonista) le llevan a considerar que toda política de reconciliación es utópica porque dirige su energía a una comunidad que todavía no existe. En efecto, se trata de lograr un nuevo nosotros donde se reconozcan los antiguos enemigos. La reconciliación depende de un momento que Schaap califica de arendtiano, es decir, un momento revolucionario que se produce cuando un pueblo se constituye. De ahí que en realidad la reconciliación más que restaurar un orden moral consiste en crear un nuevo orden político ${ }^{81}$.

Una consecuencia más de lo dicho. Solamente ese pasado reciente explica los perfiles del orden político que se forma. Por ello, se comprende la referencia de los defensores de la democracia agonista a la inexistencia de una identidad común en Sudáfrica durante el apartheid ni tampoco cuando éste termina. Sea como fuere, esa nueva identidad no podía urdirse con los valores abstractos y puramente estratégicos que ofrece el ideal deliberativo, porque tales valores (que, además, responden se quiera o no a una doctrina sobre el bien) no aportan mucho a la hora de reconstruir una fractura social consecuencia de un pasado trágico. $\mathrm{Y}$, aunque no sean equiparables en intensidad al caso sudafricano, todo proceso fundacional está igualmente enfrentado a algún pasado trágico en la medida en que es transicional. La identidad de las comunidades políticas se formula siempre precisamente en relación a ese pasado y exige por tanto bienes concretos, comunes por supuesto, que propongan una forma completa de plenitud humana en lo político. De ahí que los procesos de justicia transicional permiten, a mi juicio, apuntar que la comunidad política proporciona una identidad que concreta de modo fecundo la identidad humana ${ }^{82}$. En el fondo, el ideal deliberativo responde a una concepción de lo político como mero instrumento para buscar en lo privado la propia identidad y de ahí que se formule en términos de pura estrategia. Pero en los momentos verdaderamente importantes de las comunidades políticas, en las situaciones originarias, fundacionales, que se manifiestan en los procesos de transición esos valores se muestran insuficientes aunque sólo sea porque no guardan relación con el pasado trágico y no son capaces, en consecuencia, de crear una identidad a la altura de las expectativas de los miembros de dichas comunidades.

Andrew Schaap, “Agonism in divided societies”, op. cit., pp. 273-274.

82 Alfredo Cruz Prados, "Republicanismo y democracia liberal: dos conceptos de participación": Anuario Filosófico, vol. 36 (2003), pp. 83-109, aquí pp. 102-104. 
Tal vez por eso, Du Toit ha llamado a las comisiones de verdad proyectos histórico-fundacionales. Como tales proyectos, son comparables a la creación de una Constitución. El caso sudafricano mostraría además que la labor de una comisión de esa índole no es excluyente con la de una Asamblea o Parlamento Constituyente. Frente a quienes señalan que tal comparación tiene consecuencias negativas porque las comisiones de verdad miran al pasado y la creación de una Constitución debe mirar al futuro, du Toit señala que tales comisiones miran al pasado precisamente para crear un nuevo orden político y moral. Lógicamente, no establecen ningún marco jurídico e institucional del orden político, pero generan y consolidan una concepción de la moralidad política que está en la base de tal orden. En el caso sudafricano, su concepción moral constitutiva se encontraba ya en su propio nombre: verdad y reconciliación $^{83}$.

A la luz de todo lo expuesto, cabe concluir subrayando que todo momento transicional tiene algo de fundacional. Si el momento transicional no puede dejar al margen las concepciones comprehensivas del bien, entonces el momento fundacional del que estamos hablando (el de una transición) incorpora necesariamente concepciones comprehensivas del bien. En el proceso transicional se tocan las fibras más profundas de la propia comprensión como comunidad política. La situación recién vivida obliga a cada uno a enfrentarse con la comunidad política concreta que le ha tocado vivir en ese preciso momento y lugar, y a plantearse de modo conjunto sobre qué principios quiere constituirse, cuáles son sus bienes más preciados, cuál es el modo de mirar al pasado, quién es, en definitiva, y qué es lo que aprecia. Esto ocurre en todo proceso fundacional, pero en el caso de las transiciones se añade un componente trágico (el pasado reciente y la fractura social) que manifiesta más claramente que lo que está en juego en la propia comprensión de quiénes somos como miembros de una comunidad política concreta. Y eso que está en juego entronca, y de nuevo se ve más claramente en los procesos de transición por esa necesidad ineludible de enfrentarse a un pasado trágico, directamente con la propia consideración del propio yo y de la propia condición como ser humano.

83 Andre du Toit, "The Moral Foundation of the South African TRC. Truth as Acknowledgment and Justice as Recognition", en Robert I. Rotberg \& Dennis Thompson (eds.), Truth v. Justice: The Morality of Truth Commissions, op. cit., pp. 122-140, aquí pp. 124-126. 